\title{
Influence of Hydrostatic Pressure on Magnetic Properties of $(\mathrm{Sr}, \mathrm{La})(\mathrm{Ru}, \mathrm{Cr}, \mathrm{Mn}) \mathrm{O}_{3}$
}

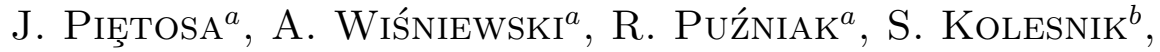 \\ M. MAJjIgA ${ }^{b}$ AND B. DABRowski ${ }^{b}$ \\ ${ }^{a}$ Institute of Physics, Polish Academy of Sciences \\ al. Lotników 32/46, 02-668 Warsaw, Poland \\ ${ }^{b}$ Physics Department, Northern Illinois University, De Kalb, IL 60115, USA
}

An influence of hydrostatic pressure, $P$, on phase transition temperature, $T_{\mathrm{C}}$, and spontaneous magnetisation, $M_{0}$, of selected perovskite ruthenates $\left(\mathrm{SrRuO}_{3}, \mathrm{La}_{0.2} \mathrm{Sr}_{0.8} \mathrm{RuO}_{3}, \mathrm{SrRu}_{0.9} \mathrm{Mn}_{0.1} \mathrm{O}_{3}\right.$, and $\left.\mathrm{SrRu}_{0.9} \mathrm{Cr}_{0.1} \mathrm{O}_{3}\right)$ was determined to $12 \mathrm{kbar}$. A decrease in $T_{\mathrm{C}}$ with pressure was found for all of the studied samples. The $M_{0}(P)$ remains unchanged for most of the samples, except for the $\mathrm{La}_{0.2} \mathrm{Sr}_{0.8} \mathrm{RuO}_{3}$ sample. The weakening of ferromagnetic interactions with increasing pressure is consistent with complex band structure effects related to the modulation of the $\mathrm{Ru}-\mathrm{O}$ hybridisation by the change of structural distortion.

PACS numbers: $75.30 .-\mathrm{m}, 75.30 . \mathrm{Kz}$

\section{Introduction}

$\mathrm{SrRuO}_{3}$, the $\mathrm{ABO}_{3}$-perovskite, is known as the only $4 d$ transition metal oxide that exhibits clear ferromagnetic (FM) properties. The ferromagnetism in $\mathrm{SrRuO}_{3}$, with $T_{\mathrm{C}}$ about $160 \mathrm{~K}$ [1], arises from a parallel alignment of magnetic moments of $4 d t_{2 \mathrm{~g}}$ electrons leading to the low-spin electronic configuration of $\mathrm{Ru}^{4+}$ ions $[2,3]$. Strong $\mathrm{Ru}_{t 2 \mathrm{~g}}-\mathrm{O}_{2 p}$ hybridisation leads to an itinerant character of ferromagnetism in this compound, evidenced by metallic conductivity, reduced magnetic moment, and lack of saturation of magnetisation in dc field of $300 \mathrm{kOe}$ $[3,4]$. Different substitutions in $\mathrm{SrRuO}_{3}$, namely, by lanthanide ions or alkaline earth ions at the A site [4-7] and $3 d$ transition metal ions at the B site [8], result in rapid changes of magnetic properties. The heterovalent $\mathrm{La}^{3+}$ substitution for $\mathrm{Sr}^{2+}$ causes a pronounced suppression of ferromagnetism by attenuation of $\mathrm{Ru}_{t 2 \mathrm{~g}}-\mathrm{O}_{2 p}$ hybridisation brought by the valence change of $\mathrm{Ru}$ and leads to antiferromagnetism 
in $\mathrm{LaRuO}_{3}[9]$. The $T_{\mathrm{C}}$ decreases to about $75 \mathrm{~K}$ for $\mathrm{La}_{0.2} \mathrm{Sr}_{0.8} \mathrm{RuO}_{3}$ [7]. The substitution of $\mathrm{Ru}$ ion by $\mathrm{Mn}^{4+}\left(d^{3}\right)$ eliminates one of the $\mathrm{Ru} t_{2 \mathrm{~g}}$ electrons, weakens the itinerant character of the $d$ electrons, and introduces antiferromagnetic (AFM) interactions. As a result ferromagnetism is rapidly suppressed to $T_{\mathrm{C}}=121 \mathrm{~K}$ for $\mathrm{SrRu}_{0.9} \mathrm{Mn}_{0.1} \mathrm{O}_{3}$ [10]. The Cr-substitution leads to unique enhancement of $T_{\mathrm{C}}$ up to $188 \mathrm{~K}$ [8] for $\mathrm{SrRu}_{0.9} \mathrm{Cr}_{0.1} \mathrm{O}_{3}$ due to possibility of electron transfer from $\mathrm{Ru}^{4+}$ to $\mathrm{Cr}^{4+}\left(d^{2}\right)$ leading to minority-band double exchange (DE) interaction [11].

The external pressure is a useful tool to tune the magnetism in these oxides by compression of the $\mathrm{A}-\mathrm{O}$ and $\mathrm{B}-\mathrm{O}$ bonds that may influence the structural distortion and consequently, the $\mathrm{Ru}-\mathrm{O}$ hybridisation as postulated by Mazin and Singh [3]. Neumeier et al. studied the pressure effect on magnetic and transport properties in perovskite ruthenate [12], showing that the value of pressure coefficient $\mathrm{d} T_{\mathrm{C}} / \mathrm{d} P$, equal to $-0.57 \mathrm{~K} / \mathrm{kbar}$, is in qualitative agreement with the predictions of the Wohlfarth model.

In this paper, we present the results of magnetic measurements performed for several substituted perovskite ruthenates under hydrostatic pressure to $12 \mathrm{kbar}$.

\section{Experimental details}

The stoichiometric $\mathrm{SrRuO}_{3}, \quad \mathrm{La}_{0.2} \mathrm{Sr}_{0.8} \mathrm{RuO}_{3}$, and the $\mathrm{SrRu}_{0.9} \mathrm{M}_{0.1} \mathrm{O}_{3}$ $(\mathrm{M}=\mathrm{Mn}$ and $\mathrm{Cr})$ samples have been prepared using standard ceramic synthesis method [13]. All the magnetic measurements were performed in the temperature range of 5-250 K at magnetic field up to $16 \mathrm{kOe}$ using a PAR 4500 vibrating sample magnetometer. A temperature dependence of magnetisation was measured using zero-field-cooling (ZFC) and field-cooling (FC) procedure, whereas $M(H)$ dependence was measured after FC in maximum applied field. For these measurements a miniature container of $\mathrm{CuBe}$ [14] with an inside diameter of $1.42 \mathrm{~mm}$ was employed as a pressure cell. A mixture of mineral oil-kerosene was used as a pressure transmitting medium. The pressure at low temperature was determined by the pressure dependence of the superconducting transition temperature of pure tin sensor placed near the sample.

\section{Results and discussion}

From the ZFC and FC magnetisation curves (Figs. 1a, 2a, 3a, 4a) it can be seen that the ferromagnetic transition temperature $T_{\mathrm{C}}$ decreases with pressure for all samples. $T_{\mathrm{C}}$ was determined from the magnetisation $M(T)$ curves as the temperature of the maximum slope of the derivative of $M(T),-\mathrm{d} M / \mathrm{d} T$. In Figs. 1b, $2 \mathrm{~b}, 3 \mathrm{~b}, 4 \mathrm{~b}$ hysteresis loops measured at $T=10 \mathrm{~K}$ are shown. All samples show the clear spontaneous FM moment $M_{0}$, which was extracted by linear extrapolation of $M(H)$ from a high-field region to $H=0$. The existence of $M_{0}$ together with a marked divergence between $M_{\mathrm{ZFC}}$ and $M_{\mathrm{FC}}$ at low temperatures confirms robust ferromagnetism below $T_{\mathrm{C}}$ reported earlier for these compounds $[2,7,8]$. The $M_{0}$ does not change under pressure for most of the samples, except $\mathrm{La}_{0.2} \mathrm{Sr}_{0.8} \mathrm{RuO}_{3}$, 

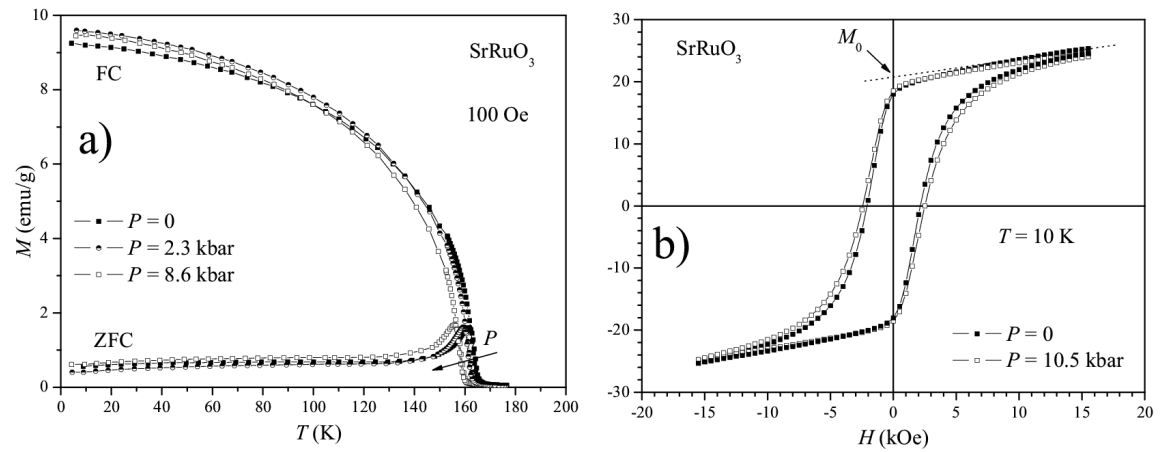

Fig. 1. (a) Temperature dependence of $M_{\mathrm{ZFC}}$ and $M_{\mathrm{FC}}$ for $\mathrm{SrRuO}_{3}$ measured at $100 \mathrm{Oe}$. (b) Magnetisation hysteresis loops for $\mathrm{SrRuO}_{3}$ at $T=10 \mathrm{~K}$.
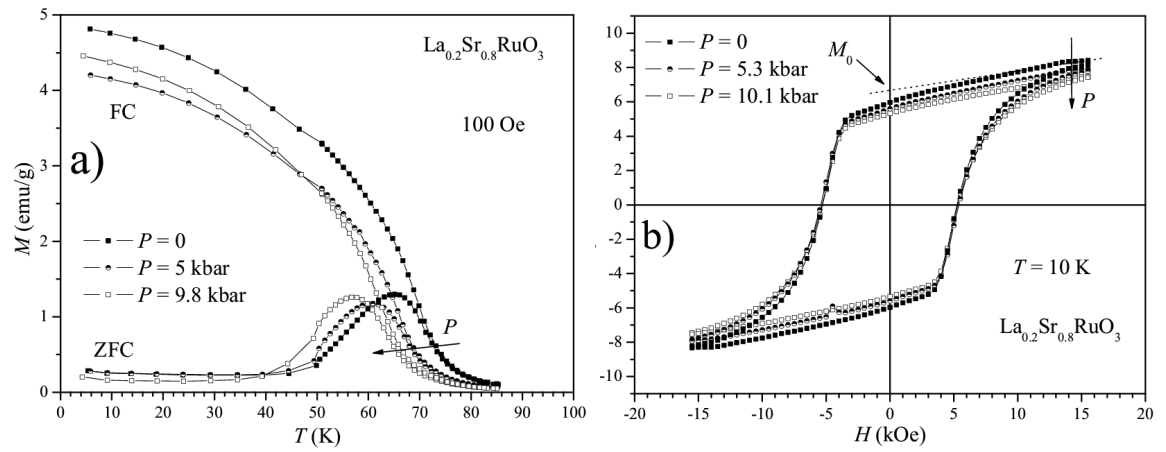

Fig. 2. (a) Temperature dependence of $M_{\mathrm{ZFC}}$ and $M_{\mathrm{FC}}$ for $\mathrm{La}_{0.2} \mathrm{Sr}_{0.8} \mathrm{RuO}_{3}$ measured at 100 Oe. (b) Magnetisation hysteresis loops for $\mathrm{La}_{0.2} \mathrm{Sr}_{0.8} \mathrm{RuO}_{3}$ at $T=10 \mathrm{~K}$.
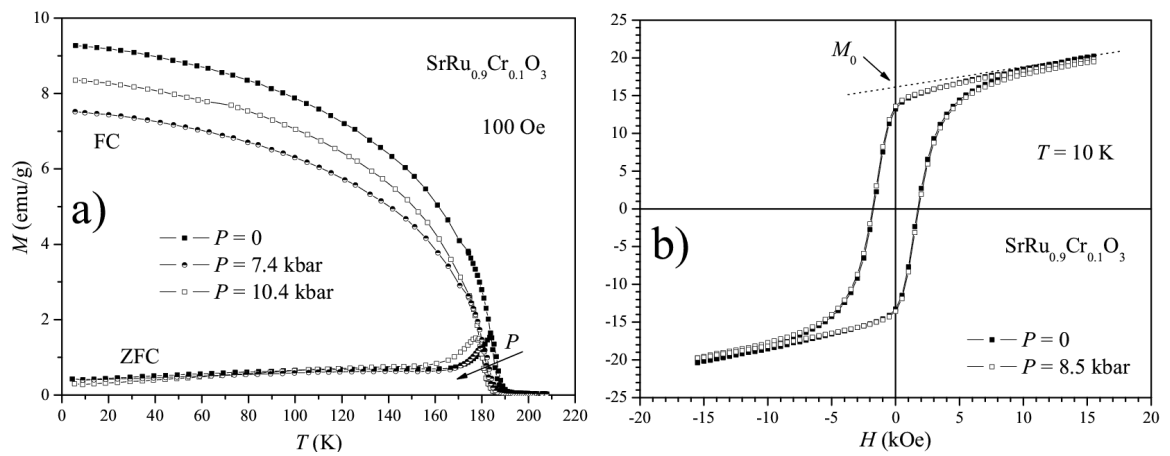

Fig. 3. (a) Temperature dependence of $M_{\mathrm{ZFC}}$ and $M_{\mathrm{FC}}$ for $\mathrm{SrRu}_{0.9} \mathrm{Cr}_{0.1} \mathrm{O}_{3}$ measured at 100 Oe. (b) Magnetisation hysteresis loops for $\mathrm{SrRu}_{0.9} \mathrm{Cr}_{0.1} \mathrm{O}_{3}$ at $T=10 \mathrm{~K}$.

where it decreases with increasing pressure. The observed changes of $T_{\mathrm{C}}$ and $M_{0}$ can be approximated by linear pressure dependence with the pressure coefficients of $\mathrm{d} T_{\mathrm{C}} / \mathrm{d} P$ and $\mathrm{d} M_{0} / \mathrm{d} P$ for $T_{\mathrm{C}}$ and $M_{0}$, respectively. 

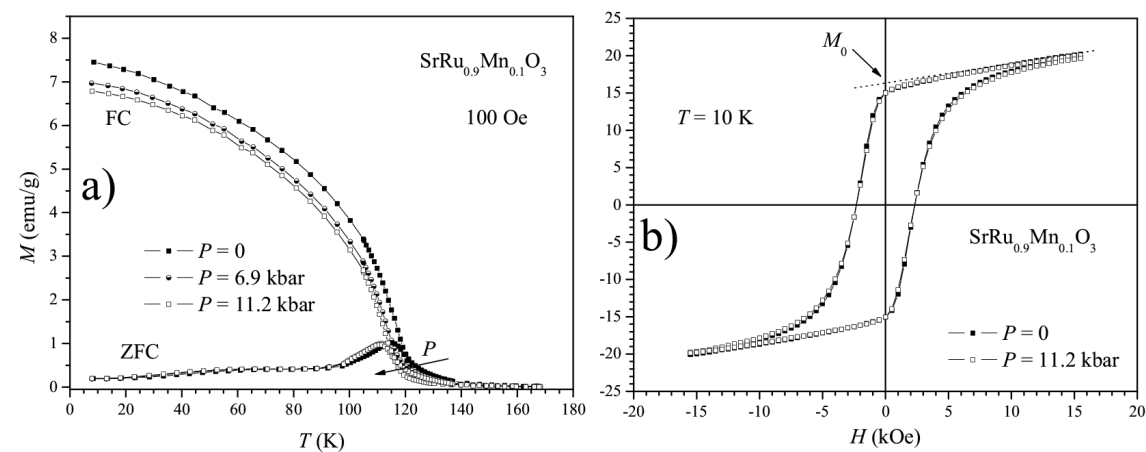

Fig. 4. (a) Temperature dependence of $M_{\mathrm{ZFC}}$ and $M_{\mathrm{FC}}$ for $\mathrm{SrRu}_{0.9} \mathrm{Mn}_{0.1} \mathrm{O}_{3}$ measured at 100 Oe. (b) Magnetisation hysteresis loops for $\mathrm{SrRu}_{0.9} \mathrm{Mn}_{0.1} \mathrm{O}_{3}$ at $T=10 \mathrm{~K}$.

The decrease in $T_{\mathrm{C}}$ with pressure for $\mathrm{SrRuO}_{3}$ was reported earlier and explained in terms of compressive strain acting on sample [11]. Our pressure coefficient $\mathrm{d} T_{\mathrm{C}} / \mathrm{d} P=-0.68 \mathrm{~K} / \mathrm{kbar}$ is in good agreement with that reported in Ref. [11] $(-0.57 \mathrm{~K} / \mathrm{kbar})$. A considerable suppression of $T_{\mathrm{C}}$ in $\mathrm{La}_{0.2} \mathrm{Sr}_{0.8} \mathrm{RuO}_{3}$, when compared to that of $\mathrm{SrRuO}_{3}$ at ambient pressure has been attributed to attenuation of $\mathrm{Ru}-\mathrm{O}$ hybridisation, caused by chemical pressure from La ion [7]. The external pressure creates further attenuation of $\mathrm{Ru}-\mathrm{O}$ hybridisation evidenced by negative pressure coefficient $(-0.67 \mathrm{~K} / \mathrm{kbar})$, in such a way that some magnetic moments become more localised and as a result the $\mathrm{Ru}_{t 2 \mathrm{~g}}-\mathrm{O}_{2 p}-\mathrm{Ru}_{t 2 \mathrm{~g}}$ AFM superexchange interactions are expected to develop at the expense of FM ones [7]. This may also cause a decrease in $M_{0}$ with pressure $\left(\mathrm{d} M_{0} / \mathrm{d} P=\right.$ $-0.07 \mathrm{emu} /(\mathrm{g}$ kbar $))$ for this sample. In the case of $\mathrm{SrRu}_{0.9} \mathrm{Cr}_{0.1} \mathrm{O}_{3}$ we observe a weakening of $\mathrm{Ru}_{t 2 \mathrm{~g}}-\mathrm{O}_{2 p}-\mathrm{Cr}_{t 2 \mathrm{~g}} \mathrm{DE}$ interactions evidenced by a decrease in $T_{\mathrm{C}}$ with increasing pressure $\left(\mathrm{d} T_{\mathrm{C}} / \mathrm{d} P=-0.53 \mathrm{~K} / \mathrm{kbar}\right)$. The spin-down electron in $\mathrm{Ru}_{t 2 \mathrm{~g}}$ band becomes more localised giving no contribution to AFM coupling of the $\mathrm{Ru}$ and $\mathrm{Cr}$ ions [10]. Therefore, $M_{0}$ remains constant with increasing pressure. For $\mathrm{SrRu}_{0.9} \mathrm{Mn}_{0.1} \mathrm{O}_{3}$ the external pressure probably affects $\mathrm{Ru}-\mathrm{O}$ hybridisation in such a way that it limits the itinerancy of the Ru $4 d$ electrons and consequently weakens the FM coupling. It is evidenced by the decrease in $T_{\mathrm{C}}$ with increasing pressure $\left(\mathrm{d} T_{\mathrm{C}} / \mathrm{d} P=-0.39 \mathrm{~K} / \mathrm{kbar}\right)$. The $M_{0}$ remains constant with increasing pressure, similar to that of $\mathrm{SrRuO}_{3}$ and $\mathrm{SrRu}_{0.9} \mathrm{Cr}_{0.1} \mathrm{O}_{3}$. For all these compounds the FM phase volume does not change under pressure. Thus, unlike substitutions on the A-site, substitutions for Ru seem to be affected in such a way that only $T_{\mathrm{C}}$ 's are changed [9].

\section{Conclusions}

An influence of hydrostatic pressure on phase transition temperature, $T_{\mathrm{C}}$, and spontaneous magnetisation, $M_{0}$, of $(\mathrm{Sr}, \mathrm{La})(\mathrm{Ru}, \mathrm{Cr}, \mathrm{Mn}) \mathrm{O}_{3}$ was investigated. We observed a decrease in $T_{\mathrm{C}}$ with pressure for all of the samples independent of 
the sign of charge doping or its affect on the FM transition. The $M_{0}(P)$ remains unchanged for most of the samples, except for the $\mathrm{La}_{0.2} \mathrm{Sr}_{0.8} \mathrm{RuO}_{3}$ sample, where $M_{0}$ decreases with pressure. The weakening of FM interactions with increasing pressure should thus be associated with a general feature of the band structure that is related to the modulation of the $\mathrm{Ru}-\mathrm{O}$ hybridisation by the change of structural distortion.

\section{Acknowledgments}

This work was supported in part by the State Committee for Scientific Research (Poland) under a research project no. 1 P03B 12330 and by the Network "New materials and sensors for optoelectronics, information technology, energetics, and medicine". Work at NIU was supported by the NSF-DMR-0302617.

\section{References}

[1] A. Callaghan, C.W. Moeller, R. Ward, Inorg. Chem. 5, 1572 (1966).

[2] L. Klein, J.S. Dodge, C.H. Ahn, J.W. Reiner, L. Mieville, T.H. Geballe, M.R. Beasley, A. Kapitulnik, J. Phys., Condens. Matter 8, 10111 (1996).

[3] I.I. Mazin, D.J. Singh, Phys. Rev. B 56, 2556 (1997).

[4] G. Cao, S. McCall, M. Shepard, J.E. Crow, R.P. Guertin, Phys. Rev. B 56, 321 (1997).

[5] A. Kanbayasi, J. Phys. Soc. Jpn. 44, 108 (1978).

[6] M.F. Da Costa, R. Greatrex, N.N. Greenwood, J. Solid State Chem. 20, 381 (1977).

[7] H. Nakatsugawa, E. Iguchi, Y. Oohara, J. Phys., Condens. Matter 14, 415 (2002).

[8] L. Pi, A. Maignan, R. Retoux, B. Raveau, J. Phys., Condens. Matter 14, 7391 (2002).

[9] R.J. Bouchard, J.F. Weiher, J. Solid State Chem. 4, 80 (1972).

[10] G. Cao, S. Chikara, X.N. Lin, E. Elhami, V. Durairaj, P. Schlottmann, Phys. Rev. B 71, 035104 (2005).

[11] B. Dabrowski, S. Kolesnik, O. Chmaissem, T. Maxwell, M. Avdeev, P.W. Barnes, J.D. Jorgensen, Phys. Rev. B 72, 054428 (2005).

[12] J.J. Neumeier, A.L. Cornelius, J.S. Schilling, Physica B 198, 324 (1994).

[13] B. Dabrowski, O. Chmaissem, P.W. Klamut, S. Kolesnik, M. Maxwell, J. Mais, Y. Ito, B.D. Armstrong, J.D. Jorgensen, S. Short, Phys. Rev. B 70, 014423 (2004).

[14] M. Baran, V. Dyakonov, L. Gladczuk, G. Levchenko, S. Piechota, H. Szymczak, Physica C 241, 383 (1995). 